\title{
B10 Complementarity determining regions (CDR) identification in Monoclonal antibodies gene sequences
}

\author{
Alexandre Bezerra Conde Figueiredoํ․, Carlos Otavio Alves Viana², \\ Márcia Arissawa ${ }^{1}$ \\ 1 Laboratório de Tecnologia de Anticorpos Monoclonais, Bio- \\ Manguinhos, Fiocruz, RJ \\ 2 Laboratório de Tecnologia Recombinante, Bio-Manguinhos, Fiocruz, \\ RJ
}

Introduction: Monoclonal antibodies (mAb) have many applications, including the use in diagnostic kits, quality control of vaccines and therapy. MAbs can be developed in the laboratory through hybridoma technology which consists in fusion and selection of B lymphocytes with tumor B-cells producing antibody of interest. Although effective, hybridoma technology has disadvantages, such as genetic instability caused by the fusion of different cells and emergence of subpopulation clones during cultivation. Each $\mathrm{mAb}$ that binds to a specific target (epitope) is derived from a $\mathrm{B}$ lymphocyte clone. The region responsible for binding and specificity of the antibody to the antigen is found in three complementarity determining regions (CDRs) in the heavy (VH) and light $(\mathrm{VL})$ chains of the $\mathrm{mAb}$ variable region. The nucleotide sequence encoding these regions is originated from recombinant genes to specific immunoglobulins in B lymphocytes, which can be identified using molecular biology techniques. Thus, the identification of CDRs is an important step to understand and preserve the genetic patrimony of mAb from LATAM's hybridoma bank. In later stages, the information will be used on new platforms of expression in order to increase productivity and efficiency in the production of antibodies.

Objective: Identify mAb CDRs from the LATAM's hybridoma bank.

Methodology: Initially we chose eight murine hybridoma from the bank, based on the service requests since last 5 years in the laboratory, among which stood out those producing $m A b$ against hepatitis $B$, yellow fever and dengue serotype 1, 23 and 4. RNA was extracted from hybridomas (RNeasy, Qiagen), and then cDNA was constructed from the reverse transcriptase reaction (SuperscriptllI, Invitrogen). The VH and $\mathrm{VL}$ regions were amplified by polymerase chain reaction (PCR), using 11 primers sense to $\mathrm{VH}$ and 9 to $\mathrm{VL}$, besides an individual antisense primer for each region. PCR products were resolved on agarose gel and used for automated sequencing (3500XL Genetic Analyzer, Life). The sequences were analyzed using the database of immunoglobulins (Vbase2 and IgBlast - NCBI) for identification and determination of the degree of CDR homology.

Results: The $\mathrm{VH}$ and $\mathrm{VL}$ regions of all hybridomas involved in this study were amplified by PCR and visualized on agarose gel $1.2 \%$, yielding a 
single band of 570 and $370 \mathrm{bp}$, respectively. The functional sequences were identified and compared using the database of immunoglobulin, obtaining $85 \%$ of homology in all of the CDR (VL and $\mathrm{VH})$ analyzed.

Conclusion: The CDRs of the six major LATAM hybridomas were identified, thus generating the possibility of population genetic homogeneity control after cloning processes, gene expression in another cell platform with higher genetic stability and, in the future, CDR manipulation by molecular biology techniques for future improvements of mAb affinity.

Keywords: Monoclonal Antibody, CDR 